\title{
Senescence of Rice Leaves XXXI. Changes of Chlorophyll, Protein, and Polyamine Contents and Ethylene Production During Senescence of a Chlorophyll-Deficient Mutant
}

\author{
Chien Teh Chen, ${ }^{1}$ Cheng Chang Li, ${ }^{2}$ and Ching Huei Kao ${ }^{1}$ \\ 'Department of Agronomy, National Taiwan University, Taipei, Taiwan; and ${ }^{2}$ Department of Agronomy, National Chung-hsing \\ University, Taichung, Taiwan, Republic of China
}

Received March 6, 1991; accepted June 25, 1991

\begin{abstract}
Leaf senescence of a chlorophylldeficient rice mutant (LT-8) was investigated. At 10 days after planting, the chlorophyll level in the third leaves of rice seedlings of the mutant was about one half that of normal leaves (Norin no. 8), whereas no difference in the protein level could be detected in the two genotypes. The protein level in leaves decreased with increasing age, and no significant difference could be detected during senescence in the two genotypes. Chlorophyll level in the normal leaves also decreased with increasing age. However, the chlorophyll level in the mutant leaves began to decrease only after more than $60 \%$ of the initial protein had been degraded. The pattern of ethylene production in the normal leaves was, in general, similar to that in the mutant leaves. Ethylene production first decreased with age, increased to a maximum at day 18 , and decreased thereafter. Both spermidine and spermine levels in the leaves of the two genotypes decreased with increasing age. The pattern of the putrescine level in the normal leaves behaved somewhat similar to that in the mutant leaves. However, during the course of senescence, the putrescine level in the mutant leaves was always higher than that in the normal leaves. The possible relationship between endogenous polyamine levels and ethylene production is discussed.
\end{abstract}

Recently, plant mutants have been widely used in the study of plant development. Leaf senescence is a syndrome, comprising a number of biochemical and physiological changes. To date, the nonyellowing genotype of meadow fescue is the only mutant used to study leaf senescence (Emyr Davies et al. 1990). LT-8 is a chlorophyll-deficient mutant of rice derived from Norin no. 8. The mutation concerns a single recessive gene, which is inherited in a Mendelian fashion and thus located at the nucleus (unpublished data). Chlorophyll loss is commonly used as a prime indicator of leaf senescence. Thus, it would be of interest to investigate the senescence syndrome of rice leaves by using this mutant.

Polyamines have recently been implicated in several facets of plant development and are particularly associated with the retardation of senescence (Evans and Malmberg 1989). The mechanism that exogenous polyamines retard leaf senescence has been proposed to be related to their possible inhibition of ethylene biosynthesis (Fuhrer et al. 1982, Shih et al. 1982). Because of this, polyamine and ethylene physiologies may be linked during leaf senescence. Thus, this article also describes the changes in free polyamine levels and ethylene production in leaves during senescence of a chlorophyll-deficient mutant. As a comparison, leaves of normal genotype, Norin no. 8 , were also included in the present investigation.

\section{Materials and Methods}

Seedlings of Oryza sativa cv. Norin no. 8 (normal) and LT-8 (mutant) were grown in hydroponic culture as described previously (Chen et al. 1990). Leaf samples (3 cm from tip) were collected from the third leaves of seedlings at various times: 10 , $12,14,16,18,20$, and 23 days after planting. For the experiments with detached leaves, the apical $3-\mathrm{cm}$ segments excised from the third leaves of 10-day-old seedlings were used. A group of 10 segments was floated in a Petri dish containing $10 \mathrm{ml}$ of distilled water. Incubation was carried out at $27^{\circ} \mathrm{C}$ in darkness.

Chlorophyll content was extracted and determined according to the method of Arnon (1949). Protein was extracted and quantitated as described previously (Kao 1980). Both chlorophyll and protein were expressed as $\mathrm{mg}$ per $\mathrm{g}$ fresh weight. 
For ethylene determination, leaf segments were placed vertically in test tubes which were closed with rubber stoppers. The ethylene in the gas phase of the enclosed tubes was determined by analysis of a 1-ml sample withdrawn with a hypodermic syringe as described elsewhere (Kao and Yang 1983). Ethylene production was expressed as $\mathrm{nl}$ per $\mathrm{g}$ fresh weight per $\mathrm{h}$.

For the extraction of free polyamines, leaf samples weighing about $125 \mathrm{mg}$ were homogenized in cold $5 \mathrm{ml}$ of perchloric acid $\left(5 \%\right.$, vol/vol), and the extracts were kept at $4^{\circ} \mathrm{C}$ for $24 \mathrm{~h}$. The homogenate was centrifuged for $20 \mathrm{~min}$ at $17,000 \mathrm{~g}$, and the clear supernatant was used for benzoylation following the method of Flores and Galston (1982). One milliliter of $2 \mathrm{~N} \mathrm{NaOH}$ was mixed with the $1 \mathrm{ml}$ supernatant. After addition of $15 \mu \mathrm{l}$ benzoyl chloride, vortexing for $10 \mathrm{~s}$, and incubation for $20 \mathrm{~min}$ at room temperature, $2.5 \mathrm{ml}$ of saturated $\mathrm{NaCl}$ was added. Benzoylpolyamines were extracted in $2.5 \mathrm{ml}$ diethyl ether. After centrifugation at $3000 \mathrm{~g}$ for $15 \mathrm{~min}, 1.5 \mathrm{ml}$ of the ether phase was collected, evaporated to dryness by vacuum concentrator, and redissolved in $100 \mu \mathrm{l}$ methanol. Polyamine standards were benzoylated in a similar way. High-performance liquid chromatography (HPLC) analysis of benzoyl-polyamines was basically performed according to the method of Slocum et al. (1989). Tenmicroliter aliquots of each redissolved sample were injected into a Waters M-6 UK Universal Liquid Chromatograph. They were eluted through a $4 \times 250 \mathrm{~mm}, 5-\mu \mathrm{m}$ particle size Cl8 reversephase column at a flow rate $1 \mathrm{ml} / \mathrm{min}$ using water (solvent $\mathrm{A}$ )/ methanol (solvent $B$ ) stepped-gradient program ( $50-65 \%$ B in 7 $\mathrm{min} ; 65-80 \% \mathrm{~B}$ in $10.5 \mathrm{~min} ; 80-100 \% \mathrm{~B}$ in $2 \mathrm{~min}$ ) followed by a column cleaning/regeneration cycle. Detection was accomplished by a Waters M481 absorbance detector at $254 \mathrm{~nm}$. Results were recorded on a Waters M470 data module and plotted as nmol per $\mathrm{g}$ fresh weight.

\section{Results}

Figure 1 shows the time courses of chlorophyll and protein levels in leaf segments excised from the third leaves of 10- to 23-day-old seedlings of normal and mutant genotypes. The protein level in leaves decreased with increasing age, and no significant difference could be detected during senescence in the two genotypes. At 10 days after planting, the chlorophyll level in the mutant leaves was about one half that of normal leaves. Chlorophyll level in the mutant leaves remained unchanged between days $10-20$ and then declined with age. In contrast to the mutant leaves, the chlorophyll level in the normal leaves decreased progressively with age. When detached leaves of both the normal and mutant rice were incubated in darkness, the decline of chlorophyll and protein levels in the mutant leaves was found to be at the same rate as that in the normal ones (Fig. 2).

The pattern of ethylene production in the normal leaves was essentially similar to that in the mutant leaves (Fig. 3). Ethylene production first decreased with age, increased to a maximum at day 18 , and then decreased again. The mutant leaves produced about $10 \%$ more ethylene than the normal ones during the period from days $10-18$.

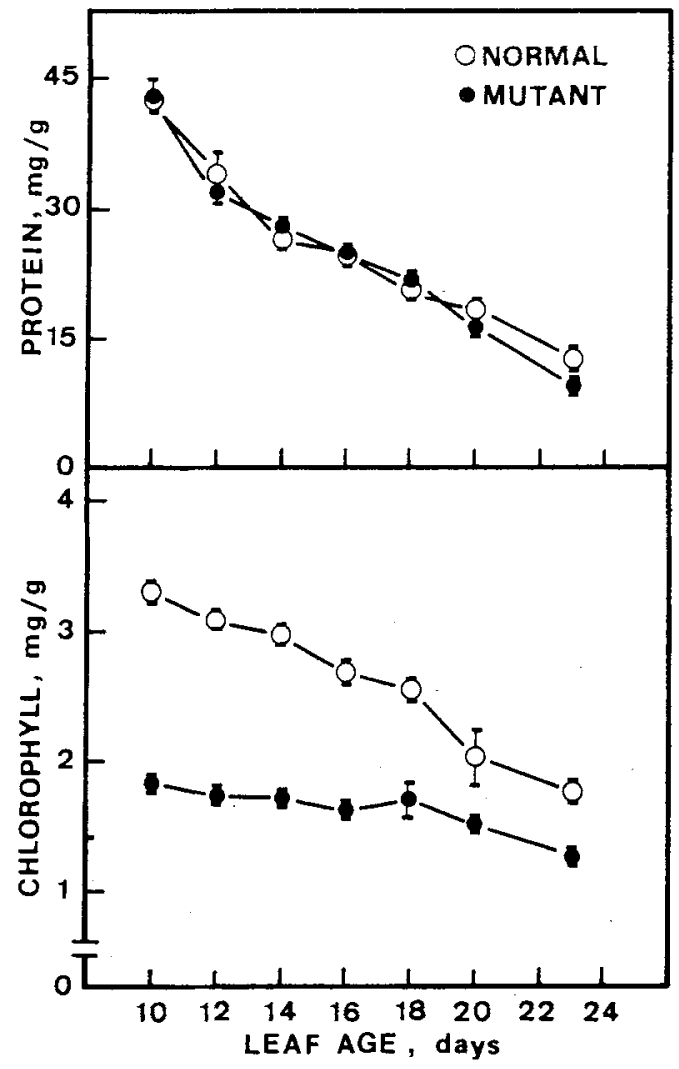

Fig. 1. Changes in chlorophyll and protein contents in normal and mutant leaves. Leaf samples were collected from the third leaves of seedlings at indicated times. Values are averages with standard errors $(\mathrm{N}=3)$.

Putrescine, spermidine, and spermine were present in both normal and mutant leaves. However, no detectable levels of cadaverine, a diamine usually found in the family Leguminosae, and diaminopropane, an oxidation product of naturally occurring polyamines, were observed in leaves of the two genotypes during senescence. Both spermidine and spermine levels in leaves decreased progressively with age in the two genotypes (Fig. 4). The change in the putrescine level in leaves during senescence showed a distinct pattern. Putrescine level in the mutant leaves increased up to day 16 , decreased at day 18 , and increased thereafter. The pattern of the putrescine level in the normal leaves behaved in a somewhat similar manner to that in mutant leaves. During the course of senescence, the putrescine level in the mutant leaves was always higher than that in the normal leaves; however, the spermidine level in the mutant leaves at days 16 and 18 was lower than that in the normal leaves.

\section{Discussion}

In senescence studies, it is commonly assumed that 


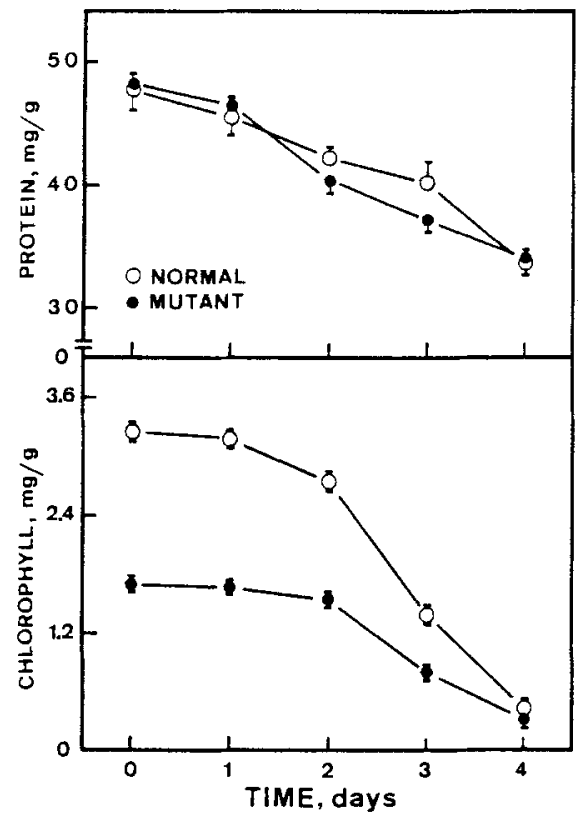

Fig. 2. Changes in chlorophyll and protein contents in detached leaves of normal and mutant rice. Detached leaves were incubated in darkness. Values are averages with standard errors $(\mathrm{N}=4)$.

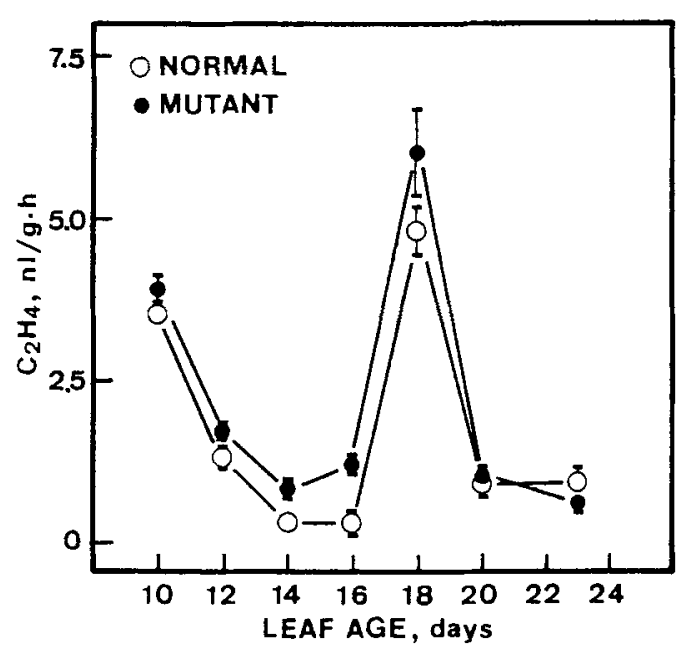

Fig. 3. Changes in ethylene production in the normal and mutant leaves. Values are averages with standard errors $(N=4)$.

chlorophyll loss is linked to protein degradation. However, the present investigation shows that the chlorophyll level in the mutant leaves began to decrease only after more than $60 \%$ of the initial protein had been degraded, suggesting that the leaf chlorophyll level is not always a reliable measure of senescence. If chlorophyll is used as the prime indicator of senescence, the leaves of the mutant

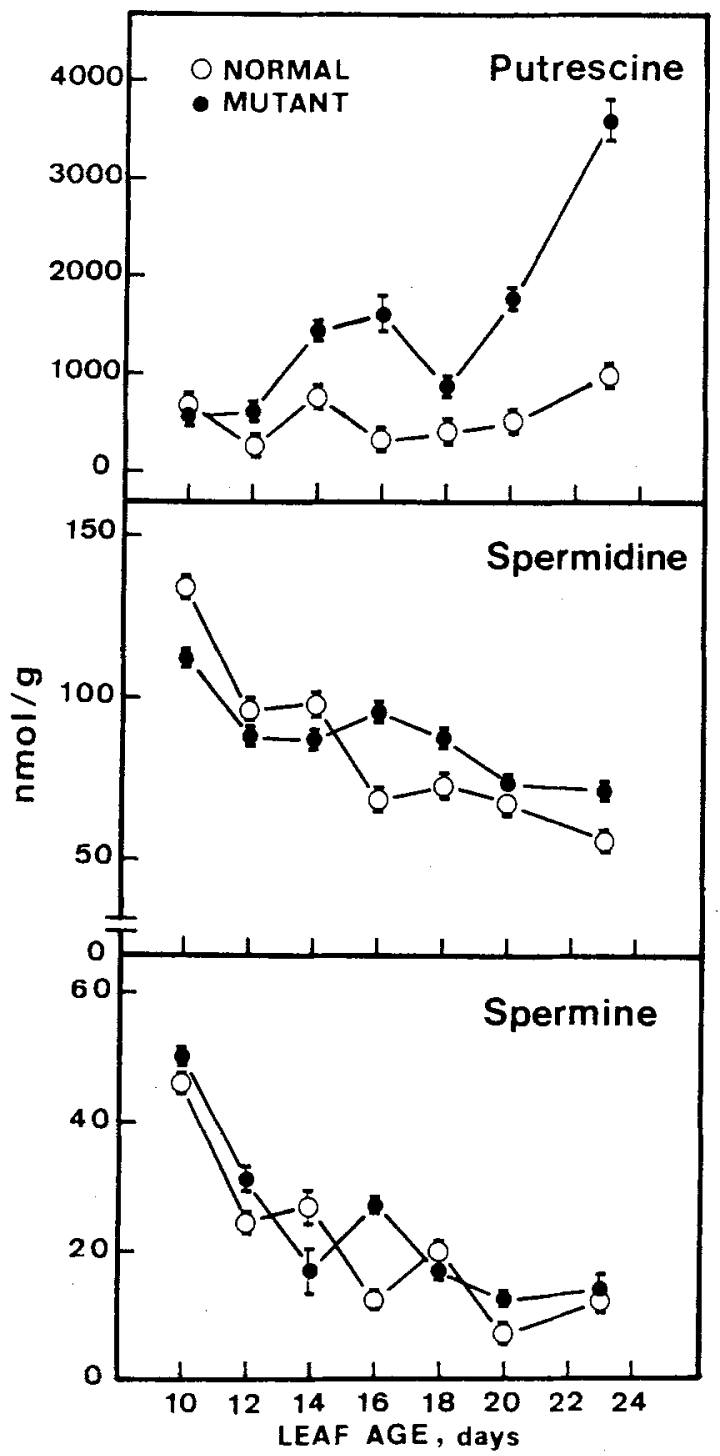

Fig. 4. Changes in polyamine contents in the normal and mutant leaves. Values are averages with standard errors $(\mathrm{N}=3)$.

would be regarded as nonsenescent or slowly senescent. However, under dark conditions, the chlorophyll level in detached leaves of the mutant decreased at a similar rate as that of detached leaves of the normal. It has been recognized for some time that excised leaves display some very substantial differences in their senescence compared with their intact counterparts (Lewington et al. 1967, Simon 1967). In view of the importance of correlative controls in senescence, it is not surprising that excision produced some big changes. Thomas and Stoddart (1975) reported that chlorophyll in detached leaves of a mutant genotype of meadow fescue remained near the initial level during a period of 6 days. They provided evidence to show that this phenomenon 
was not merely an artifact of excision, since leaves attached to the plant also retained their green color to an advanced stage of maturity.

The present investigation demonstrated that putrescine, spermidine, and spermine were present in both normal and mutant rice leaves throughout senescence. However, cadaverine and diaminopropane could not be identified in leaves of the two genotypes. Our unpublished data and previous reports showed that exogenous application of polyamines was effective in retarding chlorophyll loss in detached rice leaves (Cheng and Kao 1984, Cheng et al. 1984). If polyamines do play an important role in regulating leaf senescence of the two genotypes, then endogenous polyamine levels would be expected to decrease with increasing age. This is indeed the case with spermidine and spermine, but not putrescine. Kaur-Sawhey et al. (1982) and Srivastava et al. (1981) also found that endogenous levels of polyamines decreased with advancing leaf age.

The effect of exogenous polyamines in retarding leaf senescence is generally thought to be mediated through inhibition of ethylene production (Evans and Malmberg 1989). If this is indeed the case, then the pattern of ethylene production during senescence would be expected to be inversely correlated with that of polyamine levels. However, this is not the case in both normal and mutant leaves. Recently, we found that polyamines promoted, rather than inhibited, biosynthesis of ethylene in rice leaves (Chen et al. 1991). All these data raise the possibility that the mechanism by which polyamines regulate leaf senescence in intact rice plants may not be mediated through inhibition of ethylene production.

A dramatic decrease in ethylene production was observed at the early stage of senescence in both mutant and normal leaves. The increase of ethylene production only occurs in an advanced stage of senescence, which is too late to play a regulatory role in leaf senescence, and is probably caused by earlier events taking place in the course of senescence. Thus, the earlier indication, that senescence of detached rice leaves is directly linked to ethylene production (Kao and Yang 1983), does not hold for senescence of intact rice leaves of the two genotypes.

Of particular interest is the finding that the mutant leaves accumulated significantly higher amount of putrescine than the normal leaves during senescence. Several mutants exhibiting abnormal flower morphologies have been shown to have elevated polyamine levels (Gerats et al. 1988, Malmberg and McIndo 1983, Rastogi and Sawhney 1990). Polyamine levels in the flower have also been shown to be related to ploidy level in Nicotiana plumbagnifolia (Figueras et al. 1990). Martin-Tanguy et al. (1990) recently demonstrated an inverse relationship between polyamine levels and the degree of phenotypic alteration induced by root-inducing, left hand-transferred DNA from Agrobacterium rhizogenes. We believe that our observations provide the first evidence showing that genotypic alteration results in an elevated level of putrescine in leaf tissue.

It appears that the common precursor, S-adenosylmethionine, limits both ethylene and polyamine synthesis. At about day 18, an increase in S-adenosylmethionine permits formation of more ethylene, the preferred pathway. In the mutant, but not wild type, the extra S-adenosylmethionine is totally consumed in making the extra ethylene; this leads to the accumulation of putrescine, which requires S-adenosylmethionine to be converted to spermidine. However, the possibility of decreased putrescine metabolism and/or increased putrescine synthesis in the mutant leaves cannot be excluded. Further studies should provide valuable information on the mechanism of putrescine accumulation in the mutant leaves during senescence.

Acknowledgments. This work was financially supported by the National Science Council of the Republic of China.

\section{References}

Arnon DI (1949) Copper enzyme in isolated chloroplast polyphenooxidase in Beta vulgaris. Plant Physiol 21:1-15

Chen CT, Chou IT, Kao CH (1990) Senescence of rice leaves $\mathrm{XX}$. Changes of proton secretion during senescence. Plant Sci 66:29-34

Chen SL, Chen CT, Kao CH (1991) Polyamines promote biosynthesis of ethylene in detached rice leaves. Plant Cell Physiol (in press)

Cheng SH, Kao CH (1983) Localized effect of polyamines on chlorophyll loss. Plant Cell Physiol 24:1463-1467

Cheng SH, Shyr YY, Kao CH (1984) Senescence of rice leaves XII. Effects of 1,3-diaminopropane, spermidine and spermine. Bot Bull Academia Sinica 25:191-196

Emyr Davies TG, Thomas H, Thomas BJ, Rogers LJ (1990) Leaf senescence in a nonyellowing mutant of Festuca pratensis. Plant Physiol 93:588-595

Evans PT, Malmberg RL (1989) Do polyamines have roles in plant development? Annu Rev Plant Physiol Plant Mol Biol 40:235-269

Figueras X, Gendy CA, Pinol MT, Tran Thanh Van K, Tiburcio AF (1990) Polyamine content in relation to ploidy level and to different organs of Nicotiana plumbaginifolia. Plant Cell Physiol 31:823-828

Flores HE, Galston AW (1982) Analysis of polyamines in higher plants by high performance liquid chromatography. Plant Physiol 69:701-706 
Fuhrer J, Kaur-Sawhney R, Shih LM, Galston AW (1982) Effects of exogenous 1,3-diaminopropane and spermidine on senescence of oat leaves II. Inhibition of ethylene biosynthesis and possible mode of action. Plant Physiol 70:15971600

Gerats AGM, Kaye C, Collins C, Maimberg RL (1988) Polyamine levels in Petunia genotypes with normal and abnormal floral morphologies. Plant Physiol 86:390-393

Kao $\mathrm{CH}$ (1980) Retardation of senescence by low temperature and benzyladenins in intact primary leaves of soybean. Plant Cell Physiol 21:239-244

Kao CH, Yang SF (1983) Role of ethylene in the senescence of detached rice leaves. Plant Physiol 73:881-885

Kaur-Sawhney R, Shih LM, Flores HE, Galston AW (1982) Relation of polyamine synthesis and titer to aging and senescence in oat leaves. Plant Physiol 69:405-410

Lewington RJ, Talbot M, Simon EW (1967) The yellowing of attached and detached cucumber cotyledons. J Exp Bot $18: 526-534$

Malmberg R, McIndoo J (1983) Abnormal floral development of a tobacco mutant with elevated polyamine levels. Nature 305:623-625

Martin-Tanguy D, Tepfer D, Paynot M, Burtin D, Heisler L, Martin C (1990) Inverse relationship between polyamine levels and the degree of phenotypic alteration induced by the root-inducing, left-hand transferred DNA from Agrobacterium rhizogenes. Plant Physiol 92:912-918

Restogi R, Sawhney VK (1990) Polyamines and flower development in the male sterile stamenless-2 mutant of tomato (Lycopersicon esculentum Mill.). Plant Physiol 93:439445

Shih LM, Kaur-Sawhney R, Fuhrer J, Samanta S, Galston AW (1982) Effects of exogenous 1,3-diaminopropane and spermidine on senescence of oat leaves I. Inhibition of protease activity, ethylene production, and chlorophyll loss as related to polyamine content. Plant Physiol 70:15921596

Simon EW (1967) Types of leaf senescence. Symp Soc Exp Biol 21:215-230

Slocum RD, Flores HE, Galston AW, Weinstein LH (1989) Improved method for HPLC analysis of polyamines, agmatine and aromatic monoamines in plant tissue. Plant Physiol 89:512-517

Srivastava SK, Raj DS, Naik BI (1981) Polyamine metabolism during ageing \& senescence of pea leaves. Indian $J$ Exp Biol 19:437-440

Thomas H, Stoddart JL (1975) Separation of chlorophyll degradation from other senescence processes in leaves of a mutant genotype of meadow fescue (Festuca pratensis). Plant Physiol 56:438-441 